\title{
PSYCHOLOGICAL LITERATURE.
}

\section{NEUROLOGY.}

Die Abhangigkeit des Hirngewichtes von dem Körpergewicht und den geistigen Fähigkeiten. Отто Sxell. Arch. f. Psychiatrie, Bd. XXIII., I892, S. 436-446.

Ueber dic Abhangigkeit des Hirngewichtes von der Körpergrosse bei den Säugethieren. Eugen Dubors. Archiv f. Anthropologie, Bd. XXV., S. 1-27.

Ueber die Abhàngigkeit des Hirngewichtes von der Körpergrossse beim Mcnschen. EUgen Dubors. Archiv f. Anthropologie, Bd. XXV., 1898 .

On a Formula for Determining the Weight of the Central Nervous Systcm of the Frog from the Weight and Length of the Entire Body. H. H. Donalnson. Decennial Publications of the University of Chicago, Vol. X., rgoz. Pp. I 2.

On the Mitosis in Nerve Cells of the Cerebellar Cortex of Fetal Cats. S. HATAI. Journal of Comparative Neurology, Vol. XI., No. 4,1901 .

On the Presence of the Centrosome in Certain Nerve Cells of the White Rat. S. Hatai. Journal of Comparative Neurology, Vol. XI., rgor.

The Division of Differentiated Cells in the Central Nervous System of the White Rat. Alice Hamilton. Journal of Comparative Neurology, Vol. XI., No. 4, I901.

Observations on the Developing Neurones of the Cerebral Cortex of Fatal Cats. S. Hatai. Journal of Comparative Neurology, Vol. XII., No. 2, 1902.

Number and Size of the Spinal Ganglion Cells and Dorsal Root Fibers in the White Rat at Different Ages. S. HataI. Journal of Comparative Neurology, Vol. XII., No. 2. June, 1902. Addrcss of the President of the Anthropological Section of the British Association for the Advancement of Science. D. J. Cuxingmam. Science, N. S., Vol. XIV., Nos. $355,35^{6}$, Oct. I $\delta$ and 25,1901 .

In any animal which possesses a nervous system it should be possible to determine the amount of substance which has been differ610 
entiated into nerve tissue and to compare this amount with that of any or all the tissues that form the remainder of the body. Owing to the mechanical difficulties which stand in the way of dissecting out the entire peripheral nervous system in any animal, and the spinal cord in the larger animals, it has come about that in vertebrates the encephalon has been, for the most part, the only portion of the nervous system measured with sufficient care to permit of comparisons. Of course the weight of the encephalon is by no means a constant fraction of the weight of the entire nervous system, nor is the proportion of the active nerve substance proper--that forming the neurones as contrasted with the medullary sheaths and the nou-nervous portion of the encephalon-similar in the encephala of different species of vertebrates. Nevertheless, despite departures from uniformity and the limitations following a comparison of the encephala alone, a study of the weight relations of the encephalon in several classes of vertebrates leads to suggestive conclusions.

For more than a century, anatomists have been in search of an explanation for the observed fact that, even within the limits of a given order or genus of mammals, the weight of the encephalon did not increase in proportion to the weight of the entire animal, but was represented by a fraction which became progressively smaller as the body-weight of the species became greater. This rough statement of these relations was the best available when Snell published his observations in 1892 . For his purpose he distinguishes two relations of the nervous system-represented by the encephalon-with which variations in weight might fairly be correlated. The first of these is the relation of the encephalon to all the metabolic activities of bodyand the second, the relation of the encephalon to the psychical activities of the animal. In a general way, the larger the mass of the animal undergoing metabolic changes, the larger will be the amount of nerve tissue required, and also, within the limitations of class and order, the more complex the psychological activities of the animal the greater the mass of the encephalon.

Regarding these relations of the nervous system as factors in determining its mass, Snell designates the former as the 'somatic factor,' and the latter as the 'psychic factor.'

Beginning with the 'somatic factor,' he states the general proposition based on studies in metabolism, that the amount of metabolic change in animals of like form but different body-weight, is proportional to the extent of the body-surface, and not to the body-weight. In order to get this relation into a mathematical form, he defines 
animals of like form as those having similar geometrical figures, the surfaces of which are therefore proportional to the squares of their respective diameters, and the volumes, to the cubes. It is further assumed that the specific gravities of the several encephala to be compared are so nearly alike that the relations between their weights are identical with the relations between their volumes. Hence it is possible to translate weight relations into volume relations directly.

Under these conditions, if it is desired to compare the development of the encephalon as represented by its weight in two animals of approximately like psychical abilities and similar form, but differing in body-weight, as, for example. a mouse and a rat, it would follow, according to Snell, that their brain weight would stand in the same relation as do the areas of their bodies. Thus if a represent the body-weight of the mouse and $b$ that of the rat, and the weights as above stated stand in the same relations as the volumes of the bodies, then $\sqrt[3]{a}$, and $\sqrt{ } \bar{b}$ will give comparable diameters of the bodies and the squares of these diameters expressed thus:

$$
\left(\sqrt[3]{ }^{-} a\right)^{2} \cdot(\sqrt[3]{b})^{2}
$$

will be related as the areas of the bodies or as their surfaces. If $h$ and $h_{1}$ represent the weights of the encephala for mouse and rat in the order named, then

$$
h_{2}: h_{1}::\left(\mathfrak{3}^{\prime} \bar{a}\right)^{2}:(\sqrt[3]{b})^{2}
$$

Taking the square of the cube root of the body-weight is equivalent to raising it to the $2 / 3$ power or expressed decimally the 0.666 power.

The former may then be written $: h: h_{1}:: a^{0660}: b^{0660}$. This cxponent $(0.666)$ is thought by Snell to be probably too small for mammals, and he proposes 0.68 , which is slightly larger, as the better value for the 'somatic exponent.' In Snell's article, there is no evidence that he tested the validity of the conclusion here reached, but assuming that it was valid, he passed at once to a determination of the 'psychic factor,' or that difference in the weight of the encephalon which was to be correlated with the psychic development of the animal.

His procedure nay be exemplified as follow's: It is desired to compare the weight of the encephala of the mouse and of man. If the total weight in each case is the product of the somatic and psychic factors into the body weights, then $b$ being the body-wcight of mouse, and $c$ that of man, and $p$ and $p_{1}$, being the respective 'psychic factors,' it follows that the weight relations of the two encephala will be as $p . b^{069}: p_{1} c^{069}$. 
In any instance where the brain-weight is known, therefore, a division of the brain-weight by body-weight raised to the 0.68 power will give the number designated by Snell as the 'psychic factor.' If, now, animals for which the psychic factor has been found be arranged in a series determined by a diminishing value of the 'psychic factor,' it is found that the order in which the animals appear in this series is very close to the order in which they would be arranged were their psychical abilities determined by direct observation.

For example, by this method man leads the list with a psychic factor of 0.874 and a relative brain-weight (i.e., the relation of the weight of the encephalon to that of the body) of $1 / 35$, whereas the field mouse is down towards the end of the list with a psychic factor of only 0.045 , though the relative brain is nearly the same, being $1 / 37$.

In other words, due allowance being made for the relations of the encephalon to general metabolism, the psychical abilities of vertebrates are closely correlated with the weight of the encephalon.

This problem of the relation of the weight of the brain to the size of the body in mammals was taken up again by Eugen Dubois in I $S_{9} S$. Dubois discusses the differences in functional activity between animals of like form but differing in size, also the differences in the arrangement of the nervous system so far as they depend on size. He then proceeds to render Snell's formula more precise and to test thoroughly its applicability, using for the purpose Weber's records ( $1 S 97$ ) of the brain-and body-weights of mammals-the most reliable and complete catalogue thus far published. In taking up the subject Dubois starts with the propositions: First, that two animals of the same body-weight differ in brain-weight according to their position in the zoological scale; and second, that in the case of two animals having the same grade of brain organization, the animal having the greater body-weight will also have the greater brain weight. The weight of the encephalon depends therefore on (I) the degree of cephalization and (2) on the weight of the body. Dubois then makes a definite advance by pointing out the connection between the area of the surface of the body and the increase in the number of sensory or afferent nerve fibers by which this surface is innervated. If the arrangement of the nervous system is viewed further from the standpoint of segmentation, and each segment of the body considered as innervated by a sensori-motor mechanism, these segmental mechanisms being joined by the connecting tracts which constitute the bulk of the central nervous system, then, on comparing two animals of different bodyweights but of the same grade of organization, it is impossible to 
picture the differences as depending in the first instance on the development of the sensori-motor mechanisms for each segment, which increase in weight in proportion to the increase in the skin area to which they belong, and since the grade of the two animals compared is the same, the central tracts uniting the segmental mechanisms are also proportionately increased. Since the encephalon is in the main merely a complex of such uniting tracts, they would consequently increase in weight in proportion to the increase in the area of the body surface, and there would follow the increase in encephalic weight according to the hypothesis of Snell.

It is evident, however, that the analysis of the sensori-motor segmental mechanism can be carried much further.

In the first place it has been assumed that the constituent elements in the animals compared are alike in size, and that the larger area of the larger animal would be innervated by a proportionately larger number of fibers each having the same area in cross section as in the case of the smaller animal. Again, in the larger animal, it has been assumed that the increase in the other areas supplied by nerves is the same as that which occurs in the skin-but perhaps the point which it is most necessary to determine is whether the sensory innervation in the larger animal is of the same density as in the small. Taking the retin.l as a guide, Duhois argues that since the retina becomes relatively diminished in the larger animals, it is probable that the other sensory supply to the surface behaves in the same way, and that as a consequence, the sensory surface of the larger animals is less densely innervated than we should expect to find it by Snell's hypothesis; as the result of this, the connecting tracts in the central system would be relatively less numerous and of smaller weight and the encephalon of the larger animal therefore have a smaller weight than the theory demanded.

On the efferent side of the reflex mechanism, the relation between the increase in the weight of the muscles and the number and size of the nerves innervating them still offers a number of unsettled points. It appears probable that the increased nerve supply to the larger muscles depends rather on the relative increase in the area of the cross section of the muscles than on any other change.

However, the effects of alterations in this part of the arc are probably of least influence on the wcight of the encephalon, and a much more important factor is to be found in physical features of the cerebral cortex and the formation of gyri in the encephalon. Dubois states that in the encephalon of mammals the cortex has nearly the same 
thickness, no matter what the size of the animal. This is probably an over-statement of the small variability of this layer.

Nevertheless, it is true enough to direct attention to the source of a real difference between the brains of large and small animals having the same form, since the failure of the cortex in the larger animal to increase proportionately in thickness necessitates an extra increase in area, thus causing a folding and a formation of gyri, and a brain with gyri is smaller and weighs less than a brain composed of the same number of elements and equally complex would weigh were the cortex not folded.

The foregoing paragraphs serve to show the way in which the relations between the encephalon of the small and that of the large animal of the same zoological grade have been analyzed by Dubois. As the result of such an analysis, Dubois was led to anticipate that the weight of the encephalon would increase more slowly than the formula of Snell demanded. This Dubois tested by determining the 'somatic exponent,' or, as he prefers to name it, the 'exponent of relation,' for some seven pairs of mammals, sclected from Weber's list. Here the weight of the body as well as the weight of the encephalon is given in each case, and the value of $r$, the 'exponent of relation,' is worked out. The seven values of $r$ range between 0.5412 and $0.55_{54}$, the average of all seven being $0.5^{6}{ }_{13}$. It will be seen that this is decidedly less than the value of 0.68 finally adopted by Snell. The smaller value found by Dubois is accounted for by considering that one or all of the factors which tend to make the difference in the weight of encephala relatively smaller than that in the area of the body, have been active in these cases. In one group, namely, the bats, where the change in size is not very great and is accompanied by the least modification in the enlargement of the encephalon, the increase in the weight of encephalon has been nearly proportional to the increase in the area of the body, and the value of $r$ is 0.6649 , which is practically Snell's theoretical value. It appears from this that the enlargement of the encephalon in the larger animal is certainly modified by the conditions which have been named, and probably it is further modified by conditions touching the finer structure of the encephalon - the data for which are not yet available.

Passing next to the 'psychic factor' of Snell, we find this represented by what Dubois calls his 'exponent of cephalization,' a term much to be preferred to that of Snell.

The 'exponent of cephalization' is represented by a number which, when multiplied by the weight of the animal raised to the 
$r$ th power, gives a product which is related to another product obtained by the same process, as are the respective encephalic weights of the two animals compared. Expressing this in a formula and using the same symbols which Dubois employs, we have:

$$
E: e:: C . S^{\top}: c . s^{5}
$$

where $E$ is the weight of the encephalon in the large, and $e$ that in the small animal; $S$ and $s$, the body weights, and $C$ and $c$ the ' factors of cephalization' for the same large and small animals, the weights of whose encephala are given.

It is thus possible to arrange animals in a series according to their ' cephalization exponents,' and the position in that series is found to correspond closely with the degree of psychical development exhibited by the living animal. There are limitations to the use of the 'factor of cephalization,' however, and it must always be remembered that in the first instance it is based on weight only, while there are ways in which the complexity of the encephalon and its physiological efficiency can be altered without necessarily causing any corresponding alteration in its mass.

By this investigation Dubois has made a most noteworthy contribution to our knowledge of the comparative anatomy of the nervous system. By correlating the skin area and the nerve supply to it, and then the development of the remainder of the system in relation to this afferent division supplying the skin, the changes in the mass of the central system are anatomically linked with those occurring in the rest of the body. It may be fairly said that now we can explain why the brain in large animals increases in weight so much less rapidly than does the body.

On the way to his general conclusion the author has entered a very large number of queries concerning possible differences which may exist between the brains of different animals, and the attempt to find answers to the questions should stimulate much important work. Of course, as the amount of metabolic activity varies with the area of the body surface, there is an indirect correlation between the metabolic activity and the weight of the central system, but it would appear more probable that the increase in the weight of the nervous system in larger animals was not dependent on the fact that the metabolic changes in them were greater and needed the increase in nerve tissue to control them, but because each new area of skin required to be innervated, and with the introduction of the new afferent elements other elements in the central and efferent systems were necessarily 
added in order to complete the connections over which the additional impulses must pass. We thus come to view the increase in the mass of the central system as correlated with an increase in the number of afferent elements composing its sensory side, the increase of the other parts following in an orderly way its expansion at the periphery. Encouraged by this work on mammals, Dubois went forward to see whether an 'exponent of relation' could be determined when the brain-weights of men having different body-weights were compared. As the determination of the proper body-weight and stature depends on measurements taken on men in full health and at the prime of life, it was manifestly impossible to obtain the brain-weights of the same series. Through the kindness of his friend Herr Otto Anmon, he was able to measure four groups of young healthy men (inhabitants of Baden) who were classed as very tall, tall, medium and short. The group of very short persons was lacking. Below is given a part of his table for these four groups, each group containing to persons.

\begin{tabular}{|c|c|c|c|}
\hline Grouy & $\begin{array}{c}\text { Stature } \\
\mathrm{Cm} \\
\end{array}$ & Sitting Feight & $\begin{array}{c}\text { Body Werght. } \\
\text { Kgms }\end{array}$ \\
\hline I & 177.4 & 917 & $7^{2} 74$ \\
\hline 2 & I71 9 & 89.9 & 6749 \\
\hline 3 & 1657 & 877 & $60 \mathrm{II}$ \\
\hline 4 & 1595 & 85.05 & $555^{\circ}$ \\
\hline
\end{tabular}

In addition to the measurements exhibited above, the length and breadth of the head were taken and by the aid of formula and data derived from the results of Welcker and others, a calculation of the probable brain-weights for this series of men was made. This gave a series of four average brain-weights corresponding to the four groups, any one of which weights could be compared with all the others. Six comparisons could thus be made and the value of $r$ the 'exponent of relation' determined in each instance.

In this series it was found to range between 0.1607 and $0.397^{8}$, with an average of 0.2586 . There is evidence that the limiting values above given are excessive, and that with more numerous data the values approximate 0.25 . Dubois, therefore, takes 0.25 for the value of $r$. If, then, we compare two groups of men (in this instance, males of the same race and locality) with one another, with a view to determining their relative brain-weights we find the relation can be expressed by the formula:

$$
E: e:: S^{025}: s^{025} \text { or } \dot{V} \bar{S}: \mathfrak{V}^{-}
$$

where the brain-weight of the heavier group is represented by $E$ and 
that of the lighter group by $e$, while $S$ and $s$ represent the bodyweights of the heavier and lighter groups respectively.

The formula indicates that the increase in the brain-weights is in the same ratio as the fourth roots of the body-weights. This result is most interesting in itself, for by means of it we are able to replace the older and more general statement that among men the taller and heavier persons have on the average heavier brains, by the above formula, which expresses the law according to which the brain-weight increases. It is important to note that satisfactory data for testing this relation are extremely difficult to obtain, even under the most favorable circumstances, and for this reason it will probably be some time before it can be decided whether the same exponent of relation holds among women, or when the two sexes of the same race or different races from widely different localities are compared.

Yet, despite the limitations which are at once recognized, this determination by Dubois is an important discovery. One naturally asks why among men the increase in the weight of the brain in correlation with the body-weight, is so much slower than in the other mammals of different sizes which have been studied in connection with this problem. It can be said at once that in all probability the increase in the volume of the cranial cavity must take place according to this same law, and that, so far as the modifications of the nervous system are concerned, the larger individuals would appear to stretch the existing nerve-supply as the body became greater, rather than to add a proportional number of new elements for each new unit of area.

In this same paper Dubois examined the relation between the brain-weight and stature. A test of the data just employed slows that the groups increase in stature more rapidly than does the brain-weight. If, however, instead of the entire stature the sitting height be taken, Dubois finds that on the average the brain increases in weight in the same proportion as does the sitting height, a most simple relation, and one which Dubois regards as very important. If we may comment on this result, it would point in our opinion still more strongly to the explanation given earlier for the slow increase in brain-weight in taller and heavier men, namely, to an increase in weight due to mere passive enlargement of the central system as a result of the enlargement of the bony cavities containing it.

Working along this same general line, Donaldson has been able to obtain a simple formula which expresses for the frog the weight of the entire central nervous system as dependent on the bodyweight and length of specimens of different sizes. This work differs 
from the foregoing in that it applies to a form below the mammals, deals with the entire central nervous system-brain and cord combined -and expresses the change in the weight of this system in the growing animal.

It should be remembered, however, that the growth of the frog is different from that of the mammal, since at about 5 grams weight the frog presents those proportions of limbs and trunk which are maintained through the rest of its life. The difficulty in carrying out such an investigation lies in the determination of the normal body-weight. Not only are there here present all those difficulties which occur in the case of a mammal, but also variations in body-weight due to the amount of water, a factor which in the frog can undergo wide changes, and in addition, a distinctly marked seasonal variation in the relations between the weight of the central nervous system and that of the remainder of the body, so that for any study of this sort only mid-summer frogs are to be used, or observations on specimens taken at other seasons are to be reduced to the midsummer standard. When due care was taken to obtain correct and comparable body-weights, it was found, in the first place, that the increase in the weight of the central nervous system was nearly similar to the increase in the logarithms of the body-weights of the frogs compared; the series of logarithms did not, however, increase quite so rapidly as did the weight of the central nervous system. Another factor was therefore needed to make the two curves fit. This factor was found in the length of the frog, not applied directly, but with the value of the fourth root. The series of numbers obtained by multiplying in each instance the logarithm of the body-weight by the fourth root of the length, was found to be a nearly constant fraction of the observed weight of the central nervous system.

In the case of the bull frog the denominator of the fraction was 30, and hence multiplying in any instance by this constant would raise the number to the value of the observed weight. The formula for the bull frog is as follows:

$$
\text { C.N.S. }=(\log \cdot W \times \forall \vec{L}) C
$$

where the C.N.S. is the weight of the central nervous system in milligrams; $W$ the weight of the frog in grams, and $L$ its length in millimeters, and $C$ a constant which for the bull frog has the value of 30. A similar formula applies to the leopard frog, save that in this case the constant is 28 instead of 30 . In neither species is any difference according to sex to be observed. This indicates that during their 
life the increase in the weight of the central nervous system is progressing in a regular manner, and that, too, despite the fact that the changes leading to an increase in weight are probably somewhat different in the large and small frog.

It suggests itself that the factor depending on length may be one which represents the mere passive increase in the system, whereby it adapts itself to the larger cavities in the bigger animals and thus increases in weight without increasing in complexity. If this be true, then we see that those changes depending on increase in complexity steadily diminish as the animal becomes larger. A further analysis of this group of changes must, however, await a better knowledge of the histological modifications which are there taking place. The outcome of the investigation is that any time after it has attained a weight of 5 grams the weight of the central nervous system of either $R$. catesbrana or $R$. virescens can be determined by means of a simple formula, thus showing a regular and orderly progression in those changes which lead to an increase in the weight of this system.

As regards the early growth changes in nerve elements, Hatai has been able to show that in the cerebellar cortex of the fortal cat, the largest germinal cells present what Flemming calls a heterotypical mitosis. In these cells the number of chromosomes represented by the internodes of the segmental filaments is 16 , this being the first determination of this number in the cells of the mammalian central nervous system. These general characteristics are probably the same in the other dividing cells in the central system.

In this connection some interest attaches to those structures in the cytoplasm forming centrosome and attraction sphere. It had been thought by some that since these organs of the cell play so prominent a rôle in cell division, they would probably entirely disappear from the cell when the ability to divide was lost. On searching for the centrosome in the white rat, both young and adult, Hatai found these structures in all classes of cells in the young rat at birth and also in all classes of nerve cells in the central system of the adult except in the cells of the corpus dentatum and the efferent cells in the ventral columns of the spinal cord, where the Nissl granules were so abundant as probably to obscure them.

In general the centrosome in the young rat is more easily distin. guished than in the adult but maintains the same size, both in the different classes of cells and at different ages. In most cases the centrosome is composed of two corpuscles, and in the adult rat the attraction sphere and centrosome show changes which are interpreted 
as signs of degeneration. It is hardly necessary to add that the cells in the adult which still exhibit the centrosome and sphere, have lost all power of further division.

A good deal of interest attaches to a determination of the time at which this power to divide disappears. On this point some observations of Miss Hamilton have a direct bearing. In her investigations of the nervous system of the white rat at birth and during the first four days after birth, she in the first place finds that cell division both in the brain and spinal cord was in active progress, even in the rat four days old. At first glance this would appear to be opposed to the current statement that in man cell division in the central nervous system comes to an end shortly after the third month of fotal life. It must be remembered, however, that birth among mammals is an event quite independent of the maturity of the foetus, and does not serve as a measure of development. The rat is born in a very immature condition, and before any proper comparison can be inade between the two animals as to the time of the cessation of cell division in the nervous system, a careful determination must be made of the ages at which they pass through corresponding stages in their development, for in all probability it is the stage of development which is important.

The dividing cells in the older rats are found more and more frequently in the extra-ventricular portions of the section. Miss Hamilton's attention was largely directed to the determination of two sorts of dividing cells, the smaller giving rise to supporting elements, nonnervous in character, while the larger ones developed into neurones. In a number of instances she was able to observe mitotic changes in large cells having several branches, cells which would be classed as multipolar nerve cells. If the changes in the nucleus may be taken to indicate that such a multipolar cell is about to divide, thell division in clearly differentiated nerve cells can occur, at least in the central system of the white rat. Opposed to such a conclusion is the accepted teaching that in the developing neurone the nerve fiber process or axone is the first outgrowth to appear. For if this were true it would be hard to understand what would happen to an axone under these circumstances.

It was difficult to attempt any reconciliation betweer the current teaching of the early development of the axone and those differentiated cells showing signs of division, until evidence was brought forward for the view that in certain parts of the central nervous system, the cerebral cortex for example, it was not the axone but the dendrites which were the first to appear. Bechterew in 1899 was led to 
this view from the study of silver preparations of the developing human cortex, and Paton in 1900 reached the same conclusion as the result of his investigation of the histogenesis of the cellular elements in the cortex of the pig. Hatai has been able to corroborate and extend these observations and to show very clearly in the cerebral cortex of the fotal cat that it is not until after the dendrites are formed and until the neurones are aggregated at a dis. tance from the ventricle in the fourth and fifth cell-layers of the cerebral cortex that the axone appears.

It will be noted that these observations on the late development of the axone apply to the cerebral cortex only, and the question has not been retested since the classic observations of $\mathrm{His}$ on developing cells in the spinal cord gave rise to the current view.

Nevertheless, since it is possible that in other parts of the central system the same condition may occur, the presence of differentiated nerve cells, $i$. e., those with dendrites undergoing division, can be explained without doing violence to any of the established views, for their dendrites may develop before the axone is formed.

As is generally recognized, the growth changes in the central nervous system are the result of two different processes not strictly separated but having a very different value at different periods. In the first period, enlargement of the system is the result of the multiplication of the cell elements by cell division, while in the second period the enlargement is at first mainly, and later, entirely, due to the development of cell elements already formed. Thus in a general way, out of the total number of elements formed by the early cell division, only a fraction undergo immediate development-the completion of which, in the larger mammals, may require years-while for a time, at least, new elements continue to enter upon the changes leading to complete development. Thus while the total number of neurones, immature and mature, is constant, the number of mature neurones steadily increases at the expense of the immature group.

This is the general method according to which the human nervous system is thought to be transformed from its condition at birth to that at maturity. When this series of events is analyzed, and especially when the changes occurring during old age are included, a great number of probleins arise, each of which must be settled by a detailed investigation.

In determining the number and size of the spinal ganglion cells and dorsal root fibers in the white rat, at different ages, Hatai has dealt with one of the problems in question. The investigation takes de- 
parture from two fundamental points. First, that at and after the age (about 8-10 days) of the youngest rat examined, there is no internal evidence that any cell division takes place in the cells of the spinal ganglion, and second, that all the medullated fibers in the dorsal nerve roots of the spinal cord are outgrowths of cell bodies located in the spinal ganglia. The evidence for fibers in the dorsal nerve roots havirg a different origin is, in mammals, at present too slight to impair the validity of the second point. If these points are accepted, then from the age when the rat weighs ten grams on to maturity, the number of cell bodies should remain constant. This can be determined only by comparing corresponding spinal ganglia in different rats of different ages. In this case we should hardly expect the number of cells to be exactly constant, since individual variations must occur. A study of the number of ganglion cells in four rats, the cells in three corresponding ganglia being counted in the case of each rat, shows that the number of cells in a given spinal ganglion does not increase with age, but remains constant. If the general view previously stated be correct, namely, that the neurones of this group mature in series, so that as the animal becomes older a larger and larger number of mature elements is to be found, then we should for one thing expect the number of medullated fibers in the dorsal roots to increase. Such is found to be the case. Taking all the fibers in the dorsal nerve roots, without distinguishing those which are entirely mature from those which are still immature, Hatai finds that in a rat of 167 grams body-weight there is something more than twice the number which appears in the nerves of a rat of 10 grams body-weight. If, however, attention is directed to the fibers which are entirely mature, the rat of 167 grams has from 4 to 5 times (according to the nerve) the number found in the young rat of 10 grams. Here, for the first time in the case of a mammal, Hatai has been able to follow the slow constructional changes by which the afferent system is put into connection with the spinal cord. This is seen to be accomplished by the prolonged post-natal development of the neurones, all of which are represented in the ganglia of the youngest specimen in the series. A host of questions at once arises. Do we know that we have reached the limit of this addition of new fibers? The question can be answered only by the study of older and larger rats than that which forms the upper limit of this series. Are all the cells of the ganglia represented in the dorsal nerve root by medullated fibers? In reply to this question, Hatai can show that since in a given ganglion the number of cells is constant, while the number of fibers in the dorsal root in- 
creases, the ratio of fibers to cells must be a diminishing one, but in no case does he find less than 2.7 cells for each fiber that appears in the root. This agrees with results of other investigators who have always found more cells in the ganglion than there were fibers in the dorsal root. How far this group of neurones, which does not contribute to the dorsal root fibers, does contribute to the internal complexity of the ganglion, and how far the extra cells are both physiologically and morphologically immature, further investigation must decide.

No one can look at the growth changes here taking place without feeling that variations in the amount of exercise would modify the results, and also wondering to what extent these changes in the growing nervous system of the rat are exhibited by the growing nervous system of man.

It seems most probable that such changes occur in man, but further investigation alone can show at what period the afferent pathways represented by the spinal nerve roots become numerically complete, or the rate at which that completeness is attained. It is by studies in this field, and, so far as possible, on human material that the information can be obtained to show how far the nervous system is growing during the period of formal training, and therefore what possibilities there may be of modifying its later growth by exercise and stimulation.

From quite another side Cunningham, in his presidential address given before the Anthropological section of the British Association for the Advancement of Science at the meeting of 1901 , discusses some growth changes which affect the human encephalon as a whole. He there points out that in considering the encephalon and its relations to the cranium we must regard the encephalon as the important factor, and that by its growth the form of the bony envelope has been largely determined, thus the lofty cranial vault of the human skull is a consequence of the great development of the cerebral hemispheres in man. If the cranium in its various modifications can be looked on as the outward expression of the contained encephalon, a newer craniology may, with this idea as a guide, describe more accurately than heretofore the variations in the development of the encephalon in the different races of mankind.

In this connection Cunningham takes up certain growth changes in the cerebral hemispheres which he believes to be especially characteristic of man, and which 'unquestionably have had some influence in determining head forms.'

The primate cerebrum, in contrast to that of the lower mammals, 
is distinguished by the possession of a distinct occipital lobe and by a convolutionary design which in all but a few fundamental features is different from that of any other order of mammals. Up to the time when the occipital lobe is formed on the cerebral hemispheres-that is, the time when the cerebrum changes from the quadrupedal to the primate type, the cranial envelope is closely applied to the surface of the cerebrum. At this period it would appear that there existed an antagonism between the cerebrum and the enclosing walls, although Cunningham still considers the evidence which has led to this view as insufficient. With the development of the occipital lobe, however, these relations undergo a complete change. The cranium expands more rapidly than the cerebral mass, a considerable space filled with spongy subarachnoid tissue is formed between the two and into this mass the first convolutions of the cerebral surface are pushed out. This opportunity for free growth, however, becomes less and less as the end of the pre-natal period is approached, so that at the time of birth the gyri which have been formed are closely compacted and the cranium is so moulded over the surface of these gyri that the outline of the summits of the gyri is impressed on the inner surface of the cranial wall. Pursuing further the peculiarities of the human cerebral hemispheres, Cunningham points out that the other primates also exhibit a well-developed occipital lobe. Indeed, it appears that in man the region of the occipital has suffered a comparative restriction through the encroachment of the parietal region upon it. It is to this latter region that Cunningham especially calls attention.

The recent work of Flechsig on his association-centers, the whole literature of aphasia, as well as the studies of Rudinger on the brains of eminent persons, serve to indicate the great importance of this portion of the cerebral surface. Nevertheless, Cunningham does not find any warrant for Rüdinger's sweeping statement that the higher the mental endowment the greater is the relative extent of the superior portion of the parietal lobe. On the contrary, he finds that it is the lower part of the lobe which in man, both in the earlier development and the after-growth, shows the greater relative increase.

He doubts whether the evidence is sufficient to support the views of certain authorities that an ample development of this region is to be found in the brains of men of unusual ability, but at the same time the peculiarly generous development of it in the human cerebrum makes it a region worthy of careful study. In the further examination of the inferior parietal lobe, it is pointed out how in connection with the frontal lobe it forms the superior operculum of the insula, and how in 
this way it grows downwards to meet the inferior operculum formed by the temporal lobe and so to give rise to the Sylvian fissure. The interesting point here is the angle formed by the Sylvian fissure with a horizontal plane; this angle in the young human brain, as in the primate brain generally, is large, but in the human brain alone becomes less and less as the brain matures and thus the Sylvian fissure approaches the horizontal plane. This change in direction towards the horizontal is referred to the more rapid growth of the inferior parietal lobe in its posterior or occipital portion, this part of the operculum being more energetic in its growth than that formed by the temporal lobe. As a result, Cunningham states as his own observation the very interesting fact that in the left cerebral hemisphere the Sylvian fissure, as marked by the lower boundary of the parietal lobe, is more depressed than in the right hemisphere, thus showing an overgrowth on the left side. This overgrowth, he argues, is associated with the functional differentiation of the left hemisphere, the greater physiological importance of which, especially in connection with the faculty of speech, he considers very important. This leads to a discussion of language, and the peculiar importance of the development of it in man, with which topic the address closes.

Returning now to the statements concerning the better development of the inferior parietal lobe in the left hemisphere of man, we may be permitted to raise the general question of differences between the right and left hemisphere. As Cunningham himself states, it can be shown that the superiority of the left hemisphere depends neither on greater weight, greater convolutional complexity, better blood supply or better development (i. e., higher specific gravity) of the cerebral cortex.

This being true, then the greater growth of the inferior parietal lobe must be compensated for by a smaller development of some other part of the hemisphere. Moreover, according to Eberstaller (ISgo) the principal external portion of the Sylvian fissure is longer on the left side than on the right, and longer in the brains of women than of men. The greater length of the fissure would certainly suggest a less complete development of the opercular masses, and in so far point to a smaller rather than a greater cortical extension on the left side.

Moreover, while all that Cunningham says concerning the great significance of the cerebral mechanism for speech and for the control of the finer forms of expression is most acceptable, yet the neurones immediately concerned in the control of the muscles from the cortex are predominantly in the frontal lobe and not in the parietal, so that 
the enlargement of the parietal region is at best an associated change, and since the weight of the left hemisphere does not surpass that of the right, it is also a change which must be compensated by the smaller growth of some other point.

Henry H. Donaldson.

UNIVERSITY OF CHICAgo.

\section{PSICHOPATHOLOGY.}

Psychopathological Researches: Studies in Mental Dissociation. Boris Sidis. New York, G. E. Stechert, 1902. Large 8vo. Fp. 329 and ten plates.

In the preface to this handsomely printed volume we are informed that the publication has been made possible by the Trustees of the New York Infirmary for Women and Children (and especially by Dr. Alexander Lambert). The Psychopathic Hospital and Laboratory established by them continues the work of the former Pathological Institute of the New York State Hospitals. Dr. Sidis is the director of the Psychopathic Laboratory and in presenting this first volume of studies, announces another work to be entitled 'Principles of Psychology and Psychopathology.' While this future volume will naturally be of more direct interest to psychologists, the present series of case-records presents many starting points for suggestive psychological thought. The central doctn ine to which the several cases contribute is that of dissociation; and as a result of these experimental inquiries that conception acquires at once a more definite, a more comprehensive, and a more important significance. Unquestionably the psychology of the subconscious forms one of the notable problems of contemporary inquiry; and its illumination from the side of the abnormal has, up to the present, been the most distinctive aspect of the inquiry. Along with an appreciable volume of critical investigation and judicious generalization has been put forth a far greater mass of half-baked theories and conclusions, in which all sorts of discoveries of coexisting personalities, subliminal selfs, splintered egos, strata of the unconscious, run riot; while hypnotism is yielded as a potent weapon that opens out secrets of mind as easily as a blow with a hammer lets the milk flow out of a cocoanut. It is fortunate that Dr. Sidis has determined to take up this problem from the point of view of the psychological alienist; to apply to it the methods of inquiry suggested by psychological analysis, and to interpret the phenomena presented as extreme or aberrant forms of mental interrelations the analogues of which are to be found in normal mental functioning. 